\title{
Analysis and Management of Adverse Drug Reactions after Injection of Amphotericin B in AIDS Patients with Fungal Infection
}

\author{
Caifeng Yan ${ }^{1,2,3^{*}}$, Qing Lin ${ }^{4 *}$, Buqing Su${ }^{5}$, Xiaoye Su ${ }^{6,7}$, Hanzhen Su ${ }^{1,2,3 \#, ~ L i d a ~ M o 1,2,3 \# ~}$ \\ ${ }^{1}$ Pharmacy Department/Laboratory Department of Nanning Fourth People's Hospital, Nanning, China; ${ }^{2}$ Nanning \\ Infectious Disease Hospital Affiliated to Guangxi Medical University, Nanning, China; ${ }^{3}$ Guangxi AIDS Clinical \\ Treatment Center, Nanning, China; ${ }^{4}$ Department of Infectious Diseases, Debao People's Hospital, Debao, China; \\ ${ }^{5}$ Nursing Department of Liaoning Hospital Vocational College, Shenyang, China; ${ }^{6}$ Department of Nursing, Graduate \\ School, Eternal University of the Philippines, Las Pinas, Philippines; ${ }^{7}$ Department of Critical Medicine, Fuzhou \\ Second People's Hospital, Fuzhou, China
}

Correspondence to: Hanzhen Su,672088664@qq.com; Lida Mo, 1773398578@qq.com

Keywords: AIDS, Fungus, Amphotericin B for Injection, Drugs, Adverse Reaction

Received: January 30, $2022 \quad$ Accepted: February 20, $2022 \quad$ Published: February 23, 2022

Copyright () 2022 by author(s) and Scientific Research Publishing Inc.

This work is licensed under the Creative Commons Attribution International License (CC BY 4.0).

http://creativecommons.org/licenses/by/4.0/

\section{(c) (i) Open Access}

\section{ABSTRACT}

Objective: To understand the condition of adverse drug reactions and the treatment procedures of amphotericin B for injection in patients with AIDS complicated with fungal infection. Methods: For the research object, a retrospective analysis of adverse reactions of amphotericin B injection in 121 patients of AIDS complicated with fungal infection was reported in our hospital from October 2017 to June 2021 to observe the adverse drug reactions of patients after treatment with amphotericin B for injection, 87 cases with general degree (regarded as the general group) and 34 cases with serious degree (regarded as the serious group), and analyze the general data, medication and course of disease of the two groups for symptomatic treatment. Results: Adverse reactions such as hypokalemia, abnormal liver function, renal impairment, leucopenia and drug fever were more common in the two groups, and there were also many adverse reactions such as bone marrow suppression, rash, anemia, thrombocytopenia, nausea, vomiting, fatigue, dizziness, pruritus, angina pectoris, vertigo, abdominal pain and diarrhea. The rates of leukopenia and drug fever in general group were significantly lower than those in severe group $(P<0.05)$; in the dose of amphotericin $B$ used, the rate of using $25 \mathrm{mg}$ and more than $25 \mathrm{mg}$ in the general group was significantly lower than that in the severe group $(P<0.05)$. After symptomatic treatment, most patients have improved, and a few are unknown or have not improved. Conclusion: Amphotericin $B$ for injection has great side effects and will cause different degrees of adverse 


\section{drug reactions. The dose of amphotericin B for clinical treatment should be adjusted and disposed according to the adverse reactions to avoid serious consequences.}

\section{INTRODUCTION}

Adverse drug reactions refer to the harmful reactions of qualified drugs that have nothing to do with the purpose of medication under the normal usage and dosage. Adverse drug reactions can generally be divided into four categories: side effects, toxic reactions, allergic reactions and secondary infection (also known as double infection). Adverse reactions vary in size and strength. They can make people feel uncomfortable, make the disease worse, cause new diseases, and even kill people. How to maximize the efficacy of drugs and minimize adverse reactions is a key problem to be solved in clinical practice [1-3]. The adverse drug reactions of amphotericin B include severe toxicity, such as fever, headache, chills, nausea, vomiting, loss of appetite and other adverse reactions. Intravenous administration can cause thrombophlebitis. Nephrotoxicity is common at the binding site of the drug and intracellular sterol, and proteinuria and casturia may occur; there are also anemia, leukopenia, decreased or increased blood pressure, diplopia, peripheral neuritis, and liver damage [1-6]. However, amphotericin B belongs to polyene antifungal drugs which have been on the market for more than 50 years. It has antibacterial activity against most fungi and is cheap. It is the reference standard of antifungal activity at present; moreover, Guangxi is an economically underdeveloped region in China, and also a high incidence area of opportunistic infection of AIDS with multiple fungi. In order to understand the adverse drug reactions and disposition process of amphotericin B injection in AIDS patients with fungal infection, the adverse reactions of amphotericin B injected in 121 cases of AIDS complicated with fungal infection were analyzed and disposed.

\section{MATERIALS AND METHODS}

\subsection{Research Objects}

A total of 121 patients were reported from October 2017 to June 2021 in the Fourth People's Hospital of Nanning. There were 108 male and 13 female patients with AIDS complicated with fungal infection. The average age is $(44.23 \pm 13.04)$ years, the weight is between $29-83 \mathrm{~kg}$, and the average weight is $(51.76$ $\pm 9.40) \mathrm{kg}$; Among them, there were 45 cases of Candida infection, 37 cases of Talaromyces marneffei infection, 12 cases of Cryptococcal infection, 3 cases of Aspergillus infection and 24 cases of double or multiple fungal infection. Exclusion criteria: women during pregnancy and lactation; patients with significantly abnormal underlying liver and renal function; patients who are receiving other antifungal drugs; those whose mental state cannot cooperate with the treatment. The study protocol was reviewed by the hospital medical ethics committee, and the patient gave informed consent and the informed consent was signed.

\subsection{Diagnostic Criteria}

\subsubsection{Diagnostic Criteria for AIDS (Stage AIDS)}

Epidemiological history, HIV antibody positive, CDT +4 lymphocyte count $<350 \mathrm{ul} / 1$.

\subsubsection{Diagnostic Criteria for Fungal Infection}

There are typical clinical manifestations, and pathogens are found by collecting specimens for pathology, smear microscopy and culture at the infected site; a positive cryptococcus latex agglutination test in blood or cerebrospinal fluid is the diagnosis of cryptococcus neoformans infection.

Patients who meet the diagnostic criteria for AIDS and fungal infection at the same time can be diagnosed as AIDS with fungal infection.

\subsection{Etiological Examination}

Considering fungal infection, blood, sputum, urine, feces, throat swabs, cerebrospinal fluid, bronchial 
lavage and other tests were performed, and fungus was found or cultured in 92 cases; The other 19 cases were negative, but fungal infection was diagnosed based on medical history, symptoms, signs, and laboratory and imaging studies.

\subsection{Treatment Methods}

Dosages were given according to body weight. Amphotericin B was given at $0.1 \mathrm{mg} /(\mathrm{kg} \cdot \mathrm{d})$ for the first time, and then increased by $5-10 \mathrm{mg}$ daily to the target dose $(0.5-0.7 \mathrm{mg} / \mathrm{kg} \cdot \mathrm{d})$ according to tolerance, once a day for 12 - 30 days. During the treatment, patients' symptoms and signs were closely observed; body temperature was measured regularly; electrocardiogram was checked weekly; blood routine, urine routine, liver function, kidney function and electrolyte were checked every 3 - 5 days. If the patient's condition was stable and the tolerance was good, the laboratory examination cycle could be extended to 7 days.

\subsection{Analysis Methods}

According to the "Administrative Measures for Reporting and Monitoring of Adverse Drug Reaction" issued by the Ministry of Health Order No. 81 on May 4, 2011, patients with adverse reactions after using amphotericin B for injection were collected, and patients with general adverse reactions and severe adverse reactions were identified. For patients with adverse reactions, a general group (87 cases) and a severe group (34 cases) were set up to analyze the different types of adverse reactions in the two groups; In addition, gender, age, body weight, fungal infection type, amphotericin B dose, adverse reaction time, drug withdrawal or reduction, course of disease, conversion and other indicators were compared in order to find out the way of appropriate drug adjustment and intervention according to the severity of adverse reaction.

\subsection{Statistical Analysis}

Statistical software SPSS24.0 was used for statistical analysis. The comparison of count units was performed by chi-square test. The measurement analysis was expressed as $(x \pm S)$, and the $t$ test was used for comparison. $\mathrm{P}<0.05$ was considered statistically significant.

\section{RESULTS}

\subsection{The Adverse Drug Reactions of the Two Groups}

Among the 121 cases of AIDS complicated with fungal infection after injection of amphotericin B, 87 cases were identified as general adverse reactions, accounting for $71.90 \%$, and 34 cases of severe adverse reactions, accounting for $28.10 \%$. Some patients might have 2 or more than 2 types of adverse reactions (such as renal impairment accompanied by hypokalemia, nausea and vomiting). Among the serious adverse reactions, there were 5 cases of severe leucopenia, 2 cases of general leucopenia, 11 cases of severe hypokalemia, 5 cases of general hypokalemia, 5 cases of severe drug fever, 5 cases of severe abnormal liver function, 1 case of general abnormal liver function, 1 case of severe bone marrow suppression, 2 cases of severe anemia, 5 cases of severe renal function damage and 5 cases of general renal function damage, severe thrombocytopenia occurred in 1 case, general rash in 2 cases, nausea and vomiting in 3 cases, fatigue and dizziness in 1 case, pruritus in 1 case and angina pectoris in 1 case. Among the general adverse reactions, there were 5 cases of leucopenia, 23 cases of hypokalemia, 2 cases of drug fever, 14 cases of abnormal liver function, 1 case of bone marrow suppression, 3 cases of rash, 2 cases of aggravation of anemia symptoms, 38 cases of renal function damage, 7 cases of nausea and vomiting, 3 cases of fatigue and dizziness, 3 cases of pruritus, 1 case of vertigo, 1 case of abdominal pain and 1 case of diarrhea. See Table 1 for details.

\subsection{For the Two Groups with General Information, Medication and Outcome Etc}

In the dosage of amphotericin B used in the two groups, the rate of using $25 \mathrm{mg}$ in the general group 
was significantly higher than that in the severe group, while the rate of using more than $25 \mathrm{mg}$ in the general group was significantly lower than that in the severe group ( $\mathrm{P}=0.42$ and 0.43 , respectively); Among the types of adverse reactions, the rates of leukopenia and drug fever in the general group were significantly lower than those in the severe group (P 0.14 and 0.009 , respectively), while the rates of adverse reactions such as hypokalemia, abnormal liver function and renal function damage in the two groups were not statistically significant; In the course of disease, the rate of prolongation in the general group was significantly lower than that in the severe group $(\mathrm{P}<0.001)$; In terms of outcome, the rate of no improvement in the general group was significantly lower than that in the severe group, $\mathrm{P}=0.009$. There was no significant difference in other indexes between the two groups (Table 2).

Table 1. Adverse drug reactions of amphotericin B in 121 AIDS patients with fungal infection (cases).

\begin{tabular}{|c|c|c|c|c|}
\hline \multirow[b]{2}{*}{ Type of adverse reaction } & \multicolumn{2}{|c|}{ General group } & \multicolumn{2}{|c|}{ Severe group } \\
\hline & $\begin{array}{l}\text { Worsening } \\
\text { symptoms }\end{array}$ & $\begin{array}{c}\text { General } \\
\text { symptoms }\end{array}$ & $\begin{array}{c}\text { Severe } \\
\text { symptoms }\end{array}$ & $\begin{array}{c}\text { General } \\
\text { symptoms }\end{array}$ \\
\hline Leukopenia & 0 & 5 & 5 & 2 \\
\hline Hypokalemia & 0 & 24 & 10 & 5 \\
\hline Drug fever & 0 & 2 & 5 & 0 \\
\hline Abnormal liver function & 0 & 14 & 5 & 1 \\
\hline Myelosuppression & 0 & 1 & 1 & 0 \\
\hline Rash & 0 & 3 & 0 & 2 \\
\hline Anemia & 2 & 0 & 2 & 0 \\
\hline Renal impairment & 0 & 38 & 5 & 5 \\
\hline Thrombocytopenia & 0 & 0 & 1 & 0 \\
\hline Nausea and vomiting & 0 & 7 & 0 & 3 \\
\hline Fatigue and dizziness & 0 & 3 & 0 & 1 \\
\hline Pruritus & 0 & 3 & 0 & 1 \\
\hline Angina pectoris & 0 & 0 & 0 & 1 \\
\hline Vertigo & 0 & 1 & 0 & 0 \\
\hline Abdominal pain & 0 & 1 & 0 & 0 \\
\hline Diarrhea & 0 & 1 & 0 & 0 \\
\hline
\end{tabular}

Note: The above table shows that whether it is general adverse reactions or serious adverse reactions, there is not only one type of adverse reactions, but also several types of adverse reactions combined. There are two kinds of adverse reactions: severe symptoms and general symptoms. 
Table 2. Comparison of indexes between the general adverse reactions group and the severe adverse reactions group.

\begin{tabular}{|c|c|c|c|c|}
\hline Items & $\begin{array}{l}\text { General group } \\
\quad(87 \text { cases })\end{array}$ & $\begin{array}{l}\text { Severe group } \\
\quad(34 \text { cases })\end{array}$ & $\mathrm{X}^{2} / \mathrm{t}$ & $\mathrm{P}$ \\
\hline Gender & & & 0.425 & 0.671 \\
\hline Male (cases) & $77(88.51 \%)$ & $31(91.17 \%)$ & & \\
\hline Female (cases) & $10(11.49 \%)$ & $3(8.83 \%)$ & & \\
\hline Age (years) & $44.34 \pm 13.81$ & $43.94 \pm 10.99$ & 0.168 & 0.867 \\
\hline Weight (kg) & $51.23 \pm 9.63$ & $52.57 \pm 8.61$ & 0.745 & 0.459 \\
\hline \multicolumn{5}{|l|}{ Types of common fungal infections } \\
\hline Candida (case) & $29(33.33 \%)$ & $16(47.09 \%)$ & 1.398 & 0.162 \\
\hline Talaromyces marneffei(cases) & $30(34.48 \%)$ & $7(20.59 \%)$ & 1.485 & 0.138 \\
\hline Cryptococcus (cases) & $11(12.64 \%)$ & $1(2.94 \%)$ & 1.598 & 0.110 \\
\hline \multicolumn{5}{|l|}{ Amphotericin B dose } \\
\hline Less than $25 \mathrm{mg}$ (cases) & $5(5.75 \%)$ & $3(8.82 \%)$ & 0.610 & 0.542 \\
\hline $25 \mathrm{mg}$ (cases) & $77(88.51 \%)$ & $25(73.52 \%)$ & 2.027 & 0.043 \\
\hline More than $25 \mathrm{mg}$ (cases) & $5(5.75 \%)$ & $6(17.65 \%)$ & 2.038 & 0.042 \\
\hline \multicolumn{5}{|l|}{ Main types of adverse reactions } \\
\hline Leucopenia (cases) & $5(5.75 \%)$ & $7(20.59 \%)$ & 2.445 & 0.014 \\
\hline Drug fever (cases) & $2(2.30 \%)$ & $5(14.71 \%)$ & 2.612 & 0.009 \\
\hline Hypokalemia (cases) & $24(27.59 \%)$ & $15(44.12 \%)$ & 1.742 & 0.082 \\
\hline Abnormal liver function (cases) & $14(16.09 \%)$ & $6(17.65 \%)$ & 0.206 & 0.837 \\
\hline Impairment of renal function (cases) & $38(34.48 \%)$ & $10(29.41 \%)$ & 1.436 & 0.151 \\
\hline \multicolumn{5}{|l|}{ Time of adverse reaction after use } \\
\hline Within 1 week (cases) & $46(52.87 \%)$ & $16(47.09 \%)$ & 0.573 & 0.567 \\
\hline 1 to 2 weeks (cases) & $29(33.33 \%)$ & $13(38.23 \%)$ & 0.507 & 0.621 \\
\hline More than 2 weeks (cases) & $12(13.79 \%)$ & $5(14.71 \%)$ & 0.129 & 0.897 \\
\hline \multicolumn{5}{|l|}{ Reaction of drug withdrawal and drug reduction } \\
\hline Reduce or disappear (cases) & $56(64.37 \%)$ & $28(82.35 \%)$ & 1.922 & 0.055 \\
\hline Drug reduction without stopping (cases) & $20(22.99 \%)$ & $3(8.82 \%)$ & 1.778 & 0.075 \\
\hline Unknown (cases) & $11(12.64 \%)$ & $3(8.82 \%)$ & 0.588 & 0.556 \\
\hline
\end{tabular}




$\begin{array}{lcccc}\begin{array}{c}\text { Course of the disease } \\ \text { Not obvious (cases) }\end{array} & & 6.799 & <0.001 \\ \text { Extension (cases) } & 67(77.01 \%) & 3(8.82 \%) & & \\ \quad 20(22.99 \%) & 31(91.18 \%) & & \\ \text { Outcome } & & & & \\ \quad \text { Recovery } & 21(24.14 \%) & 8(23.53 \%) & 0.070 & 0.944 \\ \text { Improvement (cases) } & 55(63.22 \%) & 20(58.82 \%) & 0.446 & 0.646 \\ \text { No improvement (cases) } & 1(1.15 \%) & 4(11.76 \%) & 2.626 & 0.009 \\ \text { Unknown (cases) } & 10(11.49 \%) & 2(5.88 \%) & 0.924 & 0.355\end{array}$

\section{DISCUSSION}

AIDS patients are prone to opportunistic infections due to their low immunity, so common complications of fungal infection are common. To control the invasion of HIV, the first step is to treat the complications. Amphotericin B for injection is a common treatment for AIDS combined with fungal infection [7-10]. Amphotericin B for injection is suitable for the treatment of the following fungal infections: Cryptococcosis, North American blastomycosis, disseminated candidiasis, coccidioidomycosis, histoplasmosis, mucor, Rhizopus, Absidia, Mucormycosis caused by endomyces and frog fecal mold, sporotrichosis caused by Shenke sporotrichosis, aspergillosis caused by Aspergillus fumigatus, etc. [11, 12]; Due to the obvious toxicity of amphotericin $\mathrm{B}$, this product is mainly used for the diagnosis of established deep fungal infection (better if it is cultured or positive for histological examination), and patients with critical and progressive disease; The signs of clinical fungal infection are not obvious, and it is not suitable for patients with positive skin or serum test; Externally used for pigmented mycosis, skin fungal infection after burning, respiratory tract Candida, Aspergillus or cryptococcus infection, fungal corneal ulcer [13-15]; Common types of adverse reactions mainly include leucopenia, hypokalemia, drug fever, abnormal liver function, bone marrow suppression, rash, anemia, impairment of renal function, thrombocytopenia, nausea, vomiting, fatigue, dizziness, pruritus, angina pectoris, vertigo, abdominal pain, diarrhea, etc.

For severe adverse drug reactions, Order No. 81 of the Ministry of Health has made it clear that it refers to a reaction caused by the use of drugs in one of the following situations: Cause death, endanger life, cause cancer, teratogenesis or birth defect, cause significant or permanent disability of human body or organ function, cause hospitalization or prolonged hospitalization, cause other important medical events, which may occur if no treatment is given. The results of this study showed that among the 34 patients with serious ADRs of amphotericin B for injection, 34 patients showed serious symptom reactions of some type of ADRs, and were also combined with various types of ADRs with serious or general symptoms, such as severe renal dysfunction combined with general nausea, fatigue, dizziness, etc. This suggests that one or more serious symptoms and different types of adverse reactions occur in the serious adverse reactions of amphotericin B for injection, and in 87 cases of general adverse reactions of amphotericin B for injection, 87 patients showed general symptoms of certain types of adverse reactions, and there were also types of adverse reactions combined with multiple general symptoms, such as general symptoms of hypokalemia combined with general symptoms of vomiting, loss of appetite, diarrhea, etc. After comparing the types of adverse reactions between patients with general adverse reactions and patients with severe adverse reactions, it was found that the latter had a higher probability of leukopenia and drug fever, while there was no significant difference between the two in the probability of hypokalemia, abnormal liver function and renal function damage.

The results also showed that there was no significant difference between patients with general adverse 
reactions and patients with serious adverse reactions in gender, age, weight, type of fungal infection, time of adverse reactions, withdrawal and reduction of drug reactions, etc; However, patients with amphotericin B doses of $25 \mathrm{mg}$ or more were more likely to cause serious adverse effects. In view of the frequent and severe adverse reactions of amphotericin B for injection in patients with AIDS complicated by fungal infection, especially the significantly prolonged course of disease and poor prognosis in patients with severe adverse reactions which threatens their lives, so it is urgent to intervene. Amphotericin B dose and use method should be cautious; Use light proof infusion device and infusion pump to drip slowly; Strictly control the dropping speed at 15 - 20 drops/min, once a day; The initial dose is $5 \mathrm{mg}$; Increase it to the treatment dose every day and maintain it until the end of the course of treatment ( $2-4$ weeks); Then, it can be changed to oral itraconazole according to the clinical characteristics; During the process of increasing the dose, if the patient has adverse reactions such as nausea, vomiting, and fever, etc., the dose can be slowed down, reduced or administered once every other day; For patients with leukopenia, the drug is discontinued in time, and Leucogenand recombinant human granulocyte-stimulating factor are given to increase white blood cell; For patients with hypokalemia, medication is stopped in time, potassium chloride sustained-release tablets are added to strengthen potassium supplementation, and potassium chloride injection is given for symptomatic treatment (oral or intravenous potassium chloride is given for the potassium supplementation according to the severity); Patients with drug fever should stop the drug in time, and if necessary, simultaneously give ibuprofen or indomethacin suppository for anti-thermal treatment; Patients with abnormal liver function should stop taking medicine in time, and give adenyl methionine butyrate disulfonate to reduce jaundice, compound glycyrrhizin, reduced glutathione, polyene phosphoryl choline for liver protection, and magnesium isoglycyrrhizate for liver protection; Patients with myelosuppression should stop the drug in time, and be given recombinant human granulocyte-stimulating factor and blood transfusion for symptomatic treatment; Patients with rash are given dexamethasone sodium phosphate injection combined with calcium gluconate injection to enhance anti-allergy treatment; Desloratadine tablets for anti-allergy treatment; Anemia patients should stop the drug in time, and be given blood transfusion to correct anemia, intravenous potassium supplement and Shengxuebao treatment; $\mathrm{Pa}$ tients with renal function impairment should stop taking medicine in time and be given Niaoduqing granule to protect the kidneys; Patients with thrombocytopenia should stop the drug in time, give platelet transfusion, and continue to take Likejun tablets or subcutaneously inject interleukin-11 to enhance platelet therapy; Patients with nausea and vomiting should stop the drug in time, and if necessary, metoclopramide should be used for symptomatic treatment; If the patients have gastrointestinal reactions again, they should be stopped drug and continue to use omeprazole to protect the stomach; Patients with fatigue and dizziness are given sodium phosphate formate sodium chloride injection/ganciclovir antiviral therapy; patients with pruritus are given antiallergic treatment with levocetirizine tablets and desloratadine tablets, and amphotericin B for injection is discontinued while itraconazole capsule is replaced for anti infection; at the same time, reduced glutathione for injection is used to protect the liver. For other general symptoms of adverse drug reactions or combined with multiple types of adverse drug reactions, corresponding treatment interventions should be given according to the principle of symptomatic treatment.

\section{CONCLUSION}

Amphotericin B for injection has serious side effects, which can cause different degrees of adverse drug reactions, and there will be adverse drug reactions with one or more types of coexisting serious symptoms or general symptoms. The clinical treatment medication needs to be monitored regularly, and the drugs should be adjusted according to the adverse reactions to avoid serious consequences.

\section{LIMITATIONS OF THE STUDY}

The number of AIDS patients with fungal infection collected in this study is small; the experience of injecting amphotericin $B$ is insufficient; the types of adverse drug reactions are not fully grasped, and the intervention of adverse drug reactions needs to be strengthened; therefore, the research has certain limita- 
tions.

\section{ACKNOWLEDGEMENTS}

This project was approved and received strong support from the functional departments of the hospital and the help of colleagues. I would like to express my heartfelt thanks to them for their support and help! I wish them good health, smooth work and all the best.

\section{FUND PROJECT}

Scientific Research Project of Health and Family Planning Commission of Guangxi Zhuang Autonomous Region (NO. Z2016067).

\section{CONFLICTS OF INTEREST}

The authors declare no conflicts of interest regarding the publication of this paper.

\section{REFERENCES}

1. Hu, H.J. (2020) Development and Clinical Application of Amphotericin B Preparations. Exploration of Rational Drug Use in China, 17, 5-9.

2. Yang, L., Liu, J., Jiang, Y., Wang, Y.J., Xu, X.F., Gan, Z.Q., Chen, Z.L. and Peng, F.H. (2021) Efficacy and Safety of Amphotericin B in the Treatment of Cryptococcal Meningitis with Rapid Dose Increase. Chinese Journal of Neuropsychiatry, 47, 348-354.

3. Qin, D.W., Yang, M.J., Lu, P.Y. and Pan, Z.Q. (2020) The Application of Amphotericin B Rapid Escalation Therapy in AIDS Complicated with Cryptococcal Meningitis. Journal of Qiannan Nationalities Medical College, 33, 185-189.

4. Xu, Z.W., Deng, W.Q. and Liu, Y.B. (2020) A Clinical Study of Amphotericin B Combined with Flucytosine and Fluconazole in the Treatment of 45 Cases of Cryptococcal Meningitis after Non-HIV Infection and Non-Organ Transplantation. Infection and Chemotherapy in China Journal, 20, 396-400.

5. Zhang, X.Y. (2016) Clinical Treatment and Nursing Countermeasures of Amphotericin B for AIDS Patients with Cryptococcal Meningitis. Anti-Infective Pharmacy, 13, 1308-1310.

6. Liang, X.Y., Cao, Y.D., Song, Y.Q. and Ma, J. (2021) A Case of Severe Anemia, Thrombocytopenia and Hypokalemia Caused by Amphotericin B Liposome for Injection. Practical Medicine and Clinical, 24, 646-648.

7. Hu, Z.L., Wei, H.X., Chi, Y., Cheng, C., Cheng, J., Chen, C., Liu, Y. and Yang, Y.F. (2014) The Application of Amphotericin B Three-Day Boosting Therapy in AIDS Complicated with Cryptococcal Meningitis. Proceedings of the 13th National Conference on Infectious Diseases of Chinese Medical Association, Guangzhou Baiyun International Convention Center, 12-15 June 2014, 449-450.

8. Huang, H.Z. and Li, Y. (2020) Analysis of the Efficacy and Safety of Amphotericin B Combined with Conventional Western Medicine in the Treatment of AIDS Complicated with Fungal Infection. Contemporary Medicine Review, 18, 137-138.

9. Xie, J.P. and Zhou, L.L. (2015) Adverse Reactions and Nursing of Amphotericin B in the Treatment of Aids Complicated with Cryptococcal Meningitis. Contemporary Nurses (Mid-Journal), No. 4, 89-90.

10. Zhao, J.R., Rui, S.J., Peng, G.C., Zhang, E.J. and Wang, Y. (2014) Efficacy and Safety of Voriconazole Combined with Amphotericin B in Induction Therapy for Cryptococcal Meningitis in HIV-Infected Patients. Chinese Journal of Experimental and Clinical Infectious Diseases (Electronic Edition), 8, 807-811.

11. Mei, H.K., Wang, D., Bai, N., Cao, J., Tang, M.J., Jiang, X.W., Wang, J. and Wang, R. (2014) Bibliometric Analy- 
sis of Amphotericin B Nephrotoxicity. China Journal of New Drugs and Clinical Medicine, 33, 924-928.

12. Hong, Z.S., Chen, Y.S., Tian, L., Liang, J.B. and Zheng, X.B. (2015) Evaluation of the Efficacy and Adverse Reactions of Amphotericin B in the Treatment of AIDS Complicated with Fungal Infection. China Pharmacy, 26, 3238-3240.

13. He, Y.H., Tan, Q., Yang, T.T., He, S.H., Wang, Y., Zhou, R.F. and Cai, L. (2019) Efficacy and Safety of Amphotericin B in the Treatment of 124 Cases of AIDS Complicated with Cryptococcal Meningitis. Chinese Journal of Mycology, 14, 346-350.

14. Sun, L.D. and Wu, J. (2014) Talking about the Adverse Reactions and Nursing Measures of the Application of Amphotericin B in the Treatment of Blood Diseases Complicated with Fungal Infection. Contemporary Medicine Review, 12, 274-275.

15. Chen, Y.S. and Liang, J.B. (2015) Evaluation of the Efficacy and Adverse Reactions of Amphotericin B in the Treatment of Fungal Infections. Northern Pharmacy, 12, 50-52. 